\title{
ROL CONSTITUTIVO Y REVISABILIDAD DE LA LÓGICA
}

\section{Constitutive Role and Revisability of Logic}

\author{
OMAR H. VÁsquez DÁVILA ${ }^{a}$ \\ https://orcid.org/0000-0002-7054-8173 \\ ovasquezdavila@campus.ungs.edu.ar \\ ${ }^{a}$ Universidad Nacional de General Sarmiento, Los Polvorines, Argentina
}

\section{Resumen}

En este trabajo analizo la propuesta de Moretti (2016) según la cual las verdades lógicas tienen dos aspectos. Por un lado, las verdades lógicas son constitutivas del vínculo entre lenguaje y mundo, por ende, siempre es necesario algún sistema lógico. Por otro lado, las verdades de una teoría lógica específica son tan revisables como las verdades de cualquier otra teoría científica, sea formal o empírica. Propongo que dicha propuesta está relacionada inevitablemente con dos cuestiones cuyas posibles respuestas ponen en duda la posibilidad de revisar o cambiar una lógica. La primera cuestión es acerca del rasgo que distintas nociones de consecuencia comparten, el cual les permitiría desempeñar un rol constitutivo en la relación lenguaje-mundo. La segunda cuestión apunta a la necesidad de precisar los criterios que conducen a la sustitución de una lógica.

Palabras clave: Verdades lógicas; Revisionismo; Lenguaje - Mundo.

\begin{abstract}
In this work, I consider Moretti's (2016) proposal according to which logical truths consist of two aspects. On the one hand, logical truths are constitutive of the link between language and world, so it is always necessary some logical system. On the other hand, the truths of a specific logical theory are as revisable as the truths of any other scientific theory, either formal or empirical. I propose that this approach is inevitably related to two questions whose possible answers challenge the possibility of changing or revising a logic. The first question is about the feature which different notions of consequence have in common, and in which sense this feature allows them to display their constitutive role in the language-world relationship. The second question points out to the necessity of precising the criteria which led us to substitute a logic.
\end{abstract}

Key words: Logical Truths; Revisionism; Language - World. 


\section{Introducción}

Una discusión importante en la filosofía de la lógica tiene que ver con el estatus epistémico de las verdades lógicas, con decidir entre si las entendemos como verdades a priori y no revisables, o si las entendemos como verdades del mismo tipo que las verdades empíricas, y al igual que éstas, sujetas a revisión. Esta polémica puede resultar iluminadora de un problema más general como el de entender el vínculo entre lenguaje y mundo. Moretti (2016) sostiene que las verdades lógicas son condiciones de posibilidad de la posesión y del uso del lenguaje, el cual a su vez sirve para objetivar un mundo. Así, las verdades lógicas son constituyentes del discurso (lenguaje) y de aquello sobre lo que versa el discurso (el mundo).

Uno de los rasgos interesantes de esta propuesta es que las verdades lógicas, a pesar de su carácter constitutivo, son revisables empíricamente. El autor sostiene que para posibilitar un lenguaje y un mundo no es necesario un sistema lógico específico sino algún sistema lógico. Otro rasgo interesante de dicha propuesta es que, dado el rol constitutivo de la lógica en la relación lenguaje-mundo, estos ámbitos no resultan independientes de aquella. Otras alternativas que encontramos en la filosofía de la lógica entienden ya sea a las estructuras del mundo o a las del lenguaje como independientes de la lógica.

No obstante, algunas cuestiones importantes quedan abiertas y pueden representar un problema para dicha propuesta. Por ejemplo, ¿qué determina que un sistema lógico específico pueda desempeñar la tarea constitutiva necesaria para la vinculación entre lenguaje y mundo? y, vinculado con esta pregunta, ¿qué criterios justifican la elección de un sistema lógico específico? Estas preguntas surgen de cómo entiende Moretti la revisabilidad de las teorías o sistemas lógicos específicos.

Este trabajo tiene la siguiente estructura. En la sección 2 reconstruyo los rasgos esenciales de la propuesta de Moretti. Luego, en la sección 3, evalúo las posibles respuestas a las preguntas del párrafo anterior y muestro que todas ellas conducen a una visión excepcionalista de la lógica.

\section{El carácter constitutivo de la lógica}

Con el objetivo de indagar la índole del nexo entre lenguaje y mundo, Moretti (2016) considera dos enfoques que discuten acerca de cuál es el fundamento de las verdades lógicas. El primer enfoque, al cual podemos llamar metalingüistico, sostiene que las verdades lógicas en- 
cuentran su fundamento en el lenguaje. ${ }^{1}$ La fuerza de este enfoque radica en la apelación a propiedades metateóricas de la lógica clásica que permiten reemplazar la noción de verdad lógica por la de teorema. Sin embargo, se ha señalado que este reemplazo no es del todo exitoso, pues si no se recurre a nociones como las de referencia y verdad, tenemos definiciones de signos lógicos inaceptables, e.g., los llamados "casos tonk". Según Moretti, esto podría ser una razón para resistirnos a la idea de que las estructuras lógicas básicas del discurso sean independientes de las estructuras básicas del mundo.

El segundo enfoque, al cual podemos llamar no-metalingüístico, encuentra el fundamento de la verdad lógica en la estructura de la realidad. ${ }^{2}$ Este enfoque se basa en la existencia de leyes formales en el mundo, las cuales justifican las verdades lógicas. Sin embargo, Moretti señala que la comprensión de esas leyes formales del mundo parece necesitar verdades lógicas conceptualmente previas. Esto podría hacernos dudar de que las estructuras básicas del mundo sean independientes de las estructuras lógicas básicas del discurso.

Esta situación lleva a Moretti a considerar una tercera opción, la cual puede entenderse como una versión del anti-excepcionalismo de la lógica. Según esta última, las verdades lógicas no gozan de un estatus epistémico especial, sino que son revisables como las verdades de cualquier otra disciplina científica. Debido a esto, preocuparnos por la justificación de la verdad lógica es preocuparnos por la justificación de la verdad simpliciter. Así, la pregunta por el vínculo entre lenguaje y mundo remite, en esta tercera opción, a la discusión por el fundamento de la verdad oracional, entendida de manera general. $\mathrm{Al}$ respecto, como en el caso anterior, tenemos dos enfoques: uno no-metalingüístico que apela a la estructura del mundo; y otro metalingüístico que encuentra dicho fundamento en la estructura del intercambio lingüístico. Moretti nos muestra cómo puede vincularse el primer enfoque con Frege, y al segundo con Davidson. ${ }^{3}$

Brevemente, la idea de Frege es que la manera de representar la forma lógica de la oración elemental debe tener en cuenta la estructura

\footnotetext{
${ }^{1}$ Este enfoque tiene muchas versiones, entre las más emblemáticas se encuentran el convencionalismo acerca de la lógica de Carnap (1937) y el inferencialismo de Dummett (1991).

${ }^{2}$ Como representantes recientes de este enfoque tenemos a Sher (2011) y Williamson (2017).

${ }^{3}$ La única razón por la cual podemos llamar 'antiexcepcionalista' a las posturas que conforman esta tercera opción es debido a que no se seguiría de ellas necesariamente que las verdades lógicas sean algo estático e invariable.
} 
de los elementos de la realidad. La realidad se estructura a partir de la vinculación de dos tipos de entidades radicalmente distintas: objetos y conceptos. El correlato lingüístico de estas entidades, manifiestas en la forma lógica, son, respectivamente, nombres y predicados. La diferencia metafísica entre objetos y conceptos es la que posibilita su vínculo. Frege recurrió a las ideas matemáticas de función y argumento, y a la idea de saturación de una función por un argumento, para representar la unidad de la oración elemental. Sin embargo, esta manera de explicar la unidad proposicional tiene algunos problemas de expresabilidad: frases como "El concepto caballo" -debido a que ocupan el lugar de un nombre en oraciones como "El concepto caballo' es difícil de entender"- no pueden referir a conceptos. La solución de Frege consiste en señalar que esta explicación muestra lo que hace posible el significado, pero no lo dice. ${ }^{4}$

Por su parte, Davidson explica la unidad oracional a partir de la inclusión del significado de una oración en un sistema de estructuras sintácticas. Para este autor, dicho sistema es fundamental para la práctica lingüística. Desde esta perspectiva, la práctica lingüística depende de que los hablantes tengan la capacidad de interpretar las preferencias de cualquiera de dichas estructuras. Para que una estructura sintáctica adquiera significado, la comprensión de cualquier oración presupone que el hablante la considera como parte de un lenguaje común. Además, el hablante considera su acto interpretativo como parte de una práctica intersubjetiva. Moretti destaca este rasgo pragmático de la propuesta de Davidson. ${ }^{5}$

Inspirado en estas ideas Moretti construye una interesante propuesta respecto de las teorías lógicas, la cual se basa en la distinción entre aspectos constitutivos y no constitutivos del lenguaje y de la realidad. El resultado es que las teorías lógicas específicas son teorías generales sobre el mundo, empíricamente revisables como cualquier otra teoría científica. Así, no hay una lógica universalmente aceptable, pues no hay una única y completa estructura del mundo.

Si bien coincido con el resultado de la propuesta de Moretti voy a señalar, en primer lugar, algunos rasgos de su propuesta que podrían dar lugar a un enfoque excepcionalista de la lógica, es decir, a la idea de que la lógica tiene rasgos distintos del resto de teorías científicas. En particular, desde esta perspectiva, las verdades lógicas, a diferencia de otras verdades científicas, no son revisables. En segundo lugar, indagaré

${ }^{4}$ Para más detalles de este planteo véase Frege (1892) y Moretti (2014). Una crítica al planteo fregeano se encuentra en Wright (2001).

${ }^{5}$ Véase Davidson (1984). 
qué criterios para elegir una teoría lógica específica (o un grupo de ellas) se pueden desprender de la propuesta del autor de La lógica y la trama de las cosas.

\section{Principios lógicos y principios lógicos específicos}

Los enfoques acerca de la unidad proposicional se relacionan con el carácter constitutivo de los principios lógicos, según Moretti, del siguiente modo:

... tener un lenguaje requiere distinguir, al menos, hablantes, palabras y oraciones; eso supone distinguir propiedades y relaciones. No se tiene un lenguaje (ni siquiera el proposicional), no se habla, si no se distinguen las oraciones de los ruidos, ni se distinguen las oraciones entre sí. Por otra parte, el lenguaje (el ser hablante) no es posible sin conexiones necesarias entre oraciones (sin normas básicas para la emisión de oraciones) y éstas no son posibles sin algo equivalente a la distinción entre nombres y predicados. La expresión lingüística del interjuego de estos requisitos se resume en algunos principios que sistematizan el uso de términos singulares, predicados y oraciones. Se los llama principios lógicos y son responsables de la estructura elemental del discurso (el logos) y, con eso, de la estructura elemental de la realidad. (Moretti, 2016, p. 16-17)

Así, según Moretti, el rol constitutivo de los principios lógicos tiene dos aspectos. Un aspecto independiente de un mundo y un lenguaje específicos, al cual podríamos llamar aspecto no-específico. Este aspecto sólo afirma la existencia de principios lógicos, no especifica cuáles. Otro aspecto dependiente de un mundo y un lenguaje específico que podemos llamar aspecto específico. La aceptabilidad de los principios lógicos específicos es, según Moretti, "empíricamente disputable y evaluable en términos de la experiencia intersubjetiva" (Moretti, 2016, p. 19). Una postura naturalista podría entender el primer aspecto como revisable, pero Moretti propone una opción que llama trascendentalista como opción al naturalismo revisionista. Según esta opción, el aspecto no específico no clasifica como teoría, por ende, no es revisable:

el uso de cierto sistema específico de principios lógicos muestra algo, algo, cuya captación puede ayudarse diciendo que tener algún sistema de principios lógicos es constitutivo del discurso y de aquello sobre lo que el discurso versa. (Moretti, 2016, p. 21) 
En cambio, la revisabilidad sí alcanza al aspecto específico, i.e., a los principios o verdades lógicas específicos. Dado este doble carácter del rol constitutivo de la lógica, Moretti nos dice que una vez que se eligió un sistema lógico específico, debe entenderse que este sistema es revisable sólo en el sentido en que es sustituible, no en el sentido en que es refutable.

De esta reconstrucción de la propuesta de Moretti se sigue la siguiente idea que me parece al menos controversial si queremos defender una visión antiexcepcionalista de la lógica: tenemos que las teorías lógicas, al hablar de lo constitutivo, son revisables en un sentido distinto a como se entiende la revisabilidad de otras teorías científicas. Mientras que en las primeras la revisabilidad se entiende como sustitución; en las segundas, la revisabilidad puede entenderse como rechazo o refutabilidad.

Bajo esta lectura de la propuesta de Moretti, un sistema lógico específico estaría vinculado por un lado con lo constitutivo, y, por otro lado, con lo empírico. El problema que veo es que, de ser así, un sistema lógico sigue ocupando un rol privilegiado en la red de creencias. Dicho de otro modo, las teorías lógicas específicas expresan algo no revisable y no empírico: lo constitutivo. Sin embargo, aquello que es lo constitutivo del lenguaje/realidad se elige o revisa en función de su relación con lo empírico. ${ }^{6}$ Lo cual nos lleva a la siguiente pregunta: ¿Cualquier teoría lógica específica-revisable sirve para mostrar lo constitutivo? Si además tenemos en cuenta la idea de que las teorías y fenómenos empíricos difícilmente determinan qué teoría lógica elegir, ${ }^{7}$ pasamos a considerar a la lógica vigente prácticamente como irrevisable en función de lo empírico. $\mathrm{O}$, al menos, resulta difícil precisar qué criterio de sustitución elegir.

El trabajo de Moretti abre la reflexión de al menos dos cuestiones que me parecen importantes. En primer lugar, dado el rol constitutivo de la lógica, parece haber algo en cada sistema lógico admisible que determina dicho rol constitutivo. Si no son sus principios lógicos específicos, ¿qué es? En segundo lugar, ¿cuál es el criterio o el modo de llevar a cabo una sustitución de la lógica subyacente?

${ }^{6}$ Incluyo aquí como empírico también a ciertos fenómenos lingüísticos.

${ }^{7}$ Autores como Williamson (2017) sostienen que la evidencia para una teoría lógica proviene de las mejores teorías no-lógicas (principalmente no empíricas, e.g., teorías acerca de la verdad, la vaguedad, etc.). Dada una teoría, uno usa la lógica para obtener las consecuencias de dicha teoría. Uno elige una teoría, junto con su lógica, basado también en otros criterios: simplicidad, poder explicativo, etc. Para una respuesta al planteo de Williamson véase Hjortland (2019). 


\section{Moretti:}

Respecto de lo primero, tenemos las siguientes palabras de

Lo que no es sustituible es el principio constituyente que pretende expresarse en la afirmación de la existencia de verdades lógicas específicas. Esto es, para que "haya" un mundo en que pensar y un lenguaje con que hacerlo, se necesitan principios de uso de signos lógicos y relaciones de consecuencia que organicen nombres y predicados y, simultáneamente, organicen objetos y relaciones. (Moretti, 2016, p. 22)

Así, dado que los sistemas lógicos poseen principios de uso de signos lógicos y relaciones de consecuencia, resultan constitutivos para la posesión de un lenguaje y un mundo. Sin embargo, las formas de entender la consecuencia lógica pueden ser tan distintas entre sí que habría que dar cuenta de cuáles son los requisitos mínimos que debe satisfacer una relación de consecuencia lógica para desempeñar su rol constitutivo. ¿Qué sería aquello compartido por distintas nociones de consecuencia lógica que les permite desempeñar su rol constitutivo?

Con respecto a lo segundo, sostener que las verdades lógicas son sustituibles, pero no refutables, obliga a que nos preguntemos cuál es el criterio para dicha sustitución. Moretti sostiene que dicho criterio es el ajuste a la experiencia intersubjetiva. Pero este criterio también puede proponerse para el rechazo de las teorías empíricas. Si consideramos que la lógica tiene el mismo estatus epistémico que el resto de disciplinas científicas, entonces, estas últimas tampoco pueden ser rechazadas, sino solamente ser sustituidas. Por lo tanto, no sería posible rechazar una teoría, sea esta empírica o lógica.

A su vez, esto nos lleva a preguntarnos acerca de la relación entre sustituir una lógica y rechazar una teoría científica. Si fuera el caso que las teorías científicas son rechazables, pero las teorías lógicas son sólo sustituibles, tenemos dos opciones, una menos plausible que la otra. Cualquiera de ellas arroja dudas sobre la posibilidad de revisar la lógica o bien de refutar una teoría científica. Podría decirse, primera opción, que al sustituir una teoría lógica se rechazan las teorías científicas (no necesariamente empíricas) asociadas a ella. Un ejemplo prima facie favorable (a esta opción poco plausible en la práctica) sería el caso de las matemáticas. Al sustituir la lógica clásica, aquellas disciplinas sufren cambios que podríamos no desear; lo cual puede, a su vez, llevarnos a reconsiderar el cambio de la teoría lógica subyacente. Sin embargo, incluso sustituciones drásticas de la lógica subyacente -como es el caso de 
la sustitución de la lógica clásica por lógicas paraconsistentes- tratan de mantener la matemática clásica. Por lo cual, la sustitución de la lógica subyacente no implica el rechazo de la teoría científica asociada a ella.

Otra opción es decir que el rechazo de una teoría científica conlleva la sustitución de una teoría lógica. Por ejemplo, podríamos pensar, de manera algo drástica, que el desarrollo de la física cuántica ocasiona el rechazo de la de la teoría física anterior. En la propuesta de Moretti, esto no ocasionaría el rechazo de nuestra lógica subyacente sino simplemente su sustitución. Sin embargo, también sucede que la misma teoría lógica puede albergar teorías científicas en disputa. Es decir, dos teorías científicas en disputa pueden estar cerradas bajo la misma noción de consecuencia lógica.

Así, ambas opciones nos llevan a poner en duda la idea de que las teorías científicas son rechazables y las teorías lógicas son sólo sustituibles. Lo cual sugiere, contrariamente a lo que quiere sostener Moretti, que las teorías lógicas no son revisables. Esto nos lleva a considerar en qué medida el cambio científico supondría, para nuestro autor, la sustitución de la lógica subyacente.

\section{Conclusión}

He tratado de inducir la idea de que la propuesta de Moretti deja abiertas al menos dos preguntas que pueden resultar peligrosas para su planteo. En primer lugar, la pregunta acerca de qué determina que una lógica pueda llevar a cabo su rol constitutivo. En segundo lugar, la pregunta acerca de cuáles son los criterios que tenemos para la sustitución de teorías lógicas. La primera cuestión advierte la necesidad de precisar la noción de consecuencia lógica, i.e., necesitamos saber en qué sentido distintas nociones de consecuencia lógica pueden satisfacer el rol constitutivo de la lógica. Las posibles respuestas a la segunda pregunta -la que tiene que ver con los criterios para sustituir un sistema lógico- parecen apoyar la idea de que las verdades lógicas no son revisables. Todo esto indicaría que la idea no específica de una lógica constitutiva del lenguaje y el mundo, es incompatible con la visión antiexcepcionalista de las teorías lógicas.

\section{Bibliografía}

Carnap, R. (1937). The logical syntax of language. Routledge \& Kegan Paul Ltd.

Davidson, D. (1984). Inquiries into truth and interpretation. Oxford 
University Press.

Dummett, M. (1991). The logical basis of metaphysics. Harvard University Press.

Frege, G. (1974 [1892]). Escritos lógico semánticos. Técnos.

Hjortland, O. (2019). What counts as evidence for a logical theory? Australasian Journal of Logic 16(7), 250-282.

Moretti, A. (2016). La lógica y la trama de las cosas. Ideas y Valores, 65 (161), 5-22.

Moretti, A. (2014). Frege: conocimiento y lenguaje. Estudios de Epistemología, 11, 67-94.

Quine, W. V. (1970). Philosophy of logic. Oxford University Press.

Sher, G. (2011). Is logic in the mind or in the world? Synthese, 181(2), 353-365.

Williamson, T. (2017). Semantic paradoxes and abductive methdology. In B. Armour-Garb. (Ed.), Reflections on the Liar (pp. 325-346). OUP.

Wright, C. (2001). Why Frege does not deserve his grain of salt: A note on the paradox of 'the concept horse' and the ascription of Bedeutungen to predicates. In B. Hale, C. Wright (Eds.), The Reason's Proper Study: Essays towards a Neo-Fregean Philosophy of Mathematics (pp. 72-90). Oxford Scholarship. 